\title{
Optical Nonlinearity Investigation of the Novel Organic-Inorganic Hybrid Salt: 1, 1'-methylenedipyridinium pentacynidonitrosoferrate(II)pentahydrate Using $Z$-Scan System
}

\author{
M.D. Zidan ${ }^{a, *}$, M.M. Al-Ktaifani ${ }^{b}$ And A. AllahHam ${ }^{a}$ \\ ${ }^{a}$ Department of Physics, \\ ${ }^{b}$ Department of Radioisotopes, Atomic Energy Commission, P.O. Box 6091, Damascus, Syria \\ (Received August 30, 2015; revised version March 14, 2016; in final form November 3, 2016)
}

The organic-inorganic hybrid salt: $1,1^{\prime}$-methylenedipyridinium pentacynidonitrosoferrate(II)pentahydrate is synthesized and characterized by multinuclear NMR, IR, and UV-vis spectroscopies and elemental analysis. The third order nonlinear optical properties of the new complex in an aqueous solution have been studied using $Z$-scan technique. Measurements were performed for input intensities $\left(I_{0}\right)$ of 437 and $241 \mathrm{~W} / \mathrm{cm}^{2}$ for the two concentrations with a CW diode laser at $635 \mathrm{~nm}$ wavelength. The nonlinear optical absorption coefficient $(\beta)$ and nonlinear refraction $\left(n_{2}\right)$ are found at $1.28 \times 10^{-3} \mathrm{~cm} / \mathrm{W}$ and $10.17 \times 10^{-9} \mathrm{~cm}^{2} / \mathrm{W}$, respectively. The overall results suggested that better nonlinearity was observed at a higher concentration of sample. Our results suggest that this material is considered as a promising candidate for future optical device applications.

DOI: 10.12693/APhysPolA.130.1326

PACS/topics: 42.65.Ky, 42.70.Nq, 42.70.Jk, 84.30.Qi

\section{Introduction}

Nonlinear optical (NLO) materials have been extensively studied for their potential applications in the protection of human eyes and solid-state optical sensors from high intensity laser beams [1-3]. A variety of organic materials have been investigated for NLO properties and optical limiting, such as: organic molecules [4-6], organic dyes [7], metallophthalocyanine [8] and fullerenes molecules and their derivatives $[9,10]$.

Special kind of $\pi$-conjugated organometallic complexes has attracted attention as promising third-order NLO materials [11-14]. These materials, which contain transition metal ions, exhibit new properties due to the richness of the various excited states present in the system and the ability to tailor the metal-organic ligand interactions. Furthermore, the delocalization of $\pi$-electron and the transfer of electron between the metal atom and the ligands make these complexes exhibit large third order NLO coefficients.

The proposed $Z$-scan method by Sheik-Bahae et al. $[15,16]$ was applied as a standard technique for separate determination of the nonlinear changes in refractive index and absorption. This method is sensitive to all nonlinear optical mechanisms that give rise to a change of the refractive index and absorption coefficient. Although number of organo-metallic complexes have been reported [17-22], using $Z$-scan technique, the investigation of organic-inorganic hybrid salts is limited [23], especially to third order NLO properties.

\footnotetext{
*corresponding author; e-mail: pscientific@aec.org.sy
}

The aim of this article is to present the synthesis, characterization, and investigation of the third-order NLO properties of the novel organic-inorganic hybrid salt: $1,1^{\prime}$-methylenedipyridinium pentacynidonitrosoferate(II)pentahydrate in aqueous solution using $Z$-scan technique at $635 \mathrm{~nm}$ wavelength.

\section{Experimental techniques}

\subsection{Materials and methods}

All reactions and manipulations were carried out in air with reagent grade solvents. $\left[\left(\mathrm{C}_{5} \mathrm{H}_{5} \mathrm{~N}\right)_{2} \mathrm{CH}_{2}\right] \mathrm{Cl}_{2} \cdot \mathrm{H}_{2} \mathrm{O}$ was prepared according to literature method [24]. $\mathrm{Na}_{2}\left[\mathrm{Fe}(\mathrm{CN})_{5} \mathrm{NO}\right] \cdot 2 \mathrm{H}_{2} \mathrm{O}$ was purchased from Merck and used as received. The Fourier transform infrared (FTIR) spectrum was recorded on Thermo-Nicolet 6700 FTIR spectrometer taken as $\mathrm{KBr}$ disc with a resolution of $4 \mathrm{~cm}^{-1}$. ${ }^{1} \mathrm{H}$ and ${ }^{13} \mathrm{C}\left\{{ }^{1} \mathrm{H}\right\}$ nuclear magnetic resonance (NMR) spectra were acquired on a Bruker Bio spin 400 spectrometer. Microanalysis was performed using EURO EA. The ultraviolet-visible (UV-vis) absorption spectrum was recorded in the wavelength range $250-600 \mathrm{~nm}$ using UV-3101 PC Shimadzo spectrophotometer.

\subsection{Synthesis of $1,1^{\prime}$-methylenedipyridinium pentacynidonitrosoferate(II)pentahydrate}

A solution of $\left[\left(\mathrm{C}_{5} \mathrm{H}_{5} \mathrm{~N}\right)_{2} \mathrm{CH}_{2}\right] \mathrm{Cl}_{2} \cdot \mathrm{H}_{2} \mathrm{O} \quad(0.40$ g, $1.53 \mathrm{mmol})$ in $\mathrm{H}_{2} \mathrm{O}(3 \mathrm{ml})$ was added to a solution of $\mathrm{Na}_{2}\left[\mathrm{Fe}(\mathrm{CN})_{5} \mathrm{NO} \cdot 2 \mathrm{H}_{2} \mathrm{O}(0.45 \mathrm{~g}, 1.53 \mathrm{mmol})\right.$ in $\mathrm{H}_{2} \mathrm{O}$ $(3 \mathrm{ml})$ at room temperature and stirred for overnight. The mixture was then left aside for two days to give brown powder, which was separated, washed with $\mathrm{Et}_{2} \mathrm{O}$ and dried to afford an earth brown powder $(0.5 \mathrm{~g}$, yield $70 \%$, at melting point $200^{\circ} \mathrm{C}$ starts to decompose.). 


\subsection{Spectroscopic data}

The spectroscopic NMR data are the following:

- ${ }^{1} \mathrm{H}$ NMR $\left(400 \mathrm{MHz}, \mathrm{D}_{2} \mathrm{O}, 25^{\circ} \mathrm{C}\right): \delta=7.34(\mathrm{~s}, 2 \mathrm{H}$, $\left.\mathrm{CH}_{2}\right), 8.24(\mathrm{~m}, 4 \mathrm{H}, \mathrm{Py}), 8.76(\mathrm{~m}, 2 \mathrm{H}, \mathrm{Py}), 9.24(\mathrm{~m}$, $4 \mathrm{H}, \mathrm{Py})$.

- ${ }^{13} \mathrm{C}\left\{{ }^{1} \mathrm{H}\right\}$ NMR: $\delta=80.91\left(\mathrm{~s}, \mathrm{CH}_{2}\right), 132.56$ (s, Py), 148.06 (s, Py), 152.87 (s, Py), 136.91 (cis-C $\equiv \mathrm{N})$, 134.69 (trans $-\mathrm{C} \equiv \mathrm{N})$.

- $\operatorname{IR}(\mathrm{KBr}): \nu \mathrm{cm}^{-1}: 2146.1(\mathrm{C} \equiv \mathrm{N}), 1920.5(\mathrm{NO})$.

- $\mathrm{UV}\left(\mathrm{H}_{2} \mathrm{O}\right): \lambda_{\max }(\varepsilon)=218.0$ (1.014), 257.5 (0.642).

\subsection{Analytical data}

The analytical data are as follows:

- $\mathrm{C}_{16} \mathrm{H}_{22} \mathrm{~N}_{8} \mathrm{O}_{6} \mathrm{Fe}$ (487.24); C 41.04 (calc. 40.18); H 5.20 (4.64); N 23.29 (23.43).

\subsection{Z-scan measurements}

The $Z$-scan experimental setup was analogous to that described in Refs. [6, 25]. The measurements were done with linearly polarized TEM $_{00}$ Gaussian beam of a CW diode laser at maximum power of $26 \mathrm{~mW}(\lambda=635 \mathrm{~nm})$. The experimental parameters during the measurements of the samples were used as follows: the laser beam is focused by a $10 \mathrm{~cm}$ focal length lens to a waist radius $\left(\omega_{0}\right)$ of $33.5 \mu \mathrm{m}$ at the focal point. The diffraction length (the Rayleigh length) $z_{0}$ is $5.5 \mathrm{~mm}$, the radius of the aperture is $r_{\mathrm{a}}=0.5 \mathrm{~mm}$ and the radius of the laser beam waist is $\omega_{\mathrm{a}}=7 \mathrm{~mm}$ on the aperture at far field. The studied samples were dissolved in water with two concentrations of $5 \times 10^{-3} \mathrm{M}$ and $5 \times 10^{-4} \mathrm{M}$. The sample cell used in this work is a $2 \mathrm{~mm}$ thick quartz cell and the cell was held on an optical rail and translated across the focal region along the axial direction that is in the direction of the propagation of the laser beam. The power transmitted through the sample is measured as a function of the sample distance $z$ from the waist plane of the Gaussian beam. The transmission of the beam through an aperture placed in the far field is measured with a power meter (Thorlabs PM300E).

\section{Results and discussion}

\subsection{NMR, FTIR, and UV-vis characterizations}

The obtained product was purely isolated as an earth brown powder and characterized by multi-nuclear NMR, FTIR, UV-vis absorption spectra and elemental analysis (see Sect. 2). The spectroscopic data are very informative and quite consistent with the molecular structure of the product (Fig. 1).

The FTIR spectrum (Fig. 2) shows the characteristic band at $2146.3 \mathrm{~cm}^{-1}$, which is readily assigned to the CN groups [26], while the distinctive absorption band at $1920.5 \mathrm{~cm}^{-1}$ is characteristic of Fe-NO group. Furthermore, the relatively high absorption frequency of the nitrosyl group (in the range 1650-1900 $\mathrm{cm}^{-1}$ ) indicates that NO is coordinated to the Fe center in a linear geometry [27]. The ${ }^{1} \mathrm{H}$ NMR spectrum shows the expected

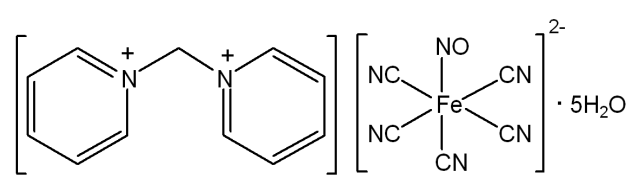

Fig. 1. Molecular structure of $1,1^{\prime}$-methylenedipyridinium pentacynidonitrosoferrate(II)pentahydrate.

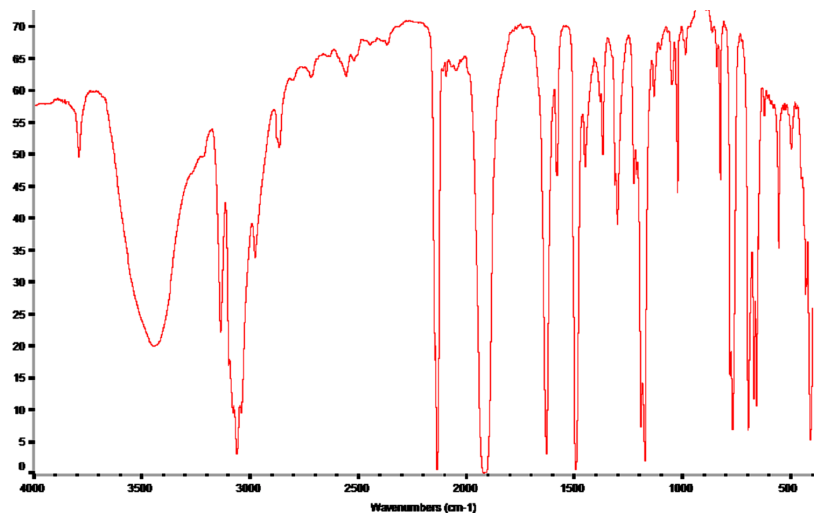

Fig. 2. FTIR spectrum of $1,1^{\prime}$-methylenedipyridinium pentacynidonitrosoferrate(II)pentahydrate (KBr disc).

four resonances for four different proton groups at 7.34 (singlet) representing the $\mathrm{CH}_{2}$ while the three resonances at $8.24,8.76$, and $9.24 \mathrm{ppm}$ correspond to three environmentally different proton groups of pyridine. The ${ }^{1} \mathrm{H}$ NMR peaks are in their expected intensity ratio. The ${ }^{13} \mathrm{C}\left\{{ }^{1} \mathrm{H}\right\}$ NMR spectrum gives six singlets for six environmentally different $\mathrm{C}$ centers, three peaks at 132.56 $\left(\mathrm{CH}_{2}\right), 148.06$, and $152.87 \mathrm{ppm}$ represent the $\mathrm{C}$ nuclei of the pyridine rings, while two peaks at 134.69 and $136.91 \mathrm{ppm}$ are observed for the two environmentally different $\mathrm{CN}$ groups. One (low intensity) $\mathrm{CN}$ group is in the trans-position, while the second (high intensity) represents four $\mathrm{C} \equiv \mathrm{N}$ groups in the cis-positions to the nitrosyl group of $\left[\mathrm{Fe}(\mathrm{CN})_{5} \mathrm{NO}\right]$. It is worth mentioning, except the extra peaks associated with $\left[\mathrm{Fe}(\mathrm{CN})_{5} \mathrm{NO}\right]$ moiety of the product, its IR, ${ }^{1} \mathrm{H}$ and ${ }^{13} \mathrm{C}\left\{{ }^{1} \mathrm{H}\right\}$ NMR spectra have the same features to their corresponding ones of the related structurally characterized organic-inorganic hybrid salts $\left[\left(\mathrm{C}_{5} \mathrm{H}_{5} \mathrm{~N}\right)_{2} \mathrm{CH}_{2}\right]\left[\mathrm{MCl}_{4}\right](\mathrm{M}=\mathrm{Zn}, \mathrm{Cd}, \mathrm{Pt}$ or $\mathrm{Cu})[28-30]$.

The UV-vis absorption spectrum of the product (Fig. 3) shows two maximum absorption peaks at $257.5(2.40)$ and $218.0(1.8) \mathrm{nm}$, which are readily attributed to $\pi \rightarrow \pi^{*}$ transition within the $1,1^{\prime}$-methylenedipyridinium dication and ligand-metal charge transfer transitions (LMCT) and/or any $d \rightarrow d^{*}$ transition of the anionic fragment, respectively.

\subsection{Nonlinear measurements}

In the present investigation, $Z$-scan experiments were performed in order to determine the nonlinear absorption coefficient $\beta$ and the nonlinear refractive index $n_{2}$ of the 1,1'-methylenedipyridinium pentacynidonitrosoferate(II)pentahydrate in aqueous solution at two concentrations of $5 \times 10^{-3} \mathrm{M}$ and $5 \times 10^{-4} \mathrm{M}$ for input intensities 


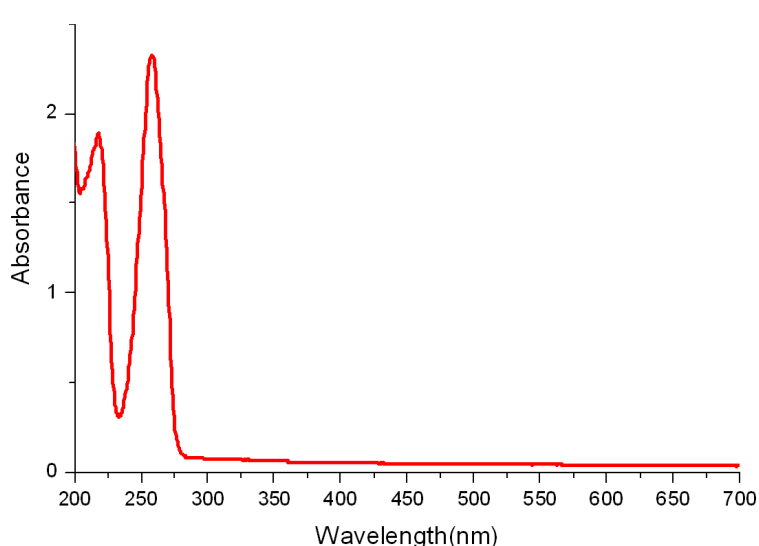

Fig. 3. UV-vis spectrum of $1,1^{\prime}$-methylenedipyridinium pentacynidonitrosoferrate(II)pentahydrate in water.

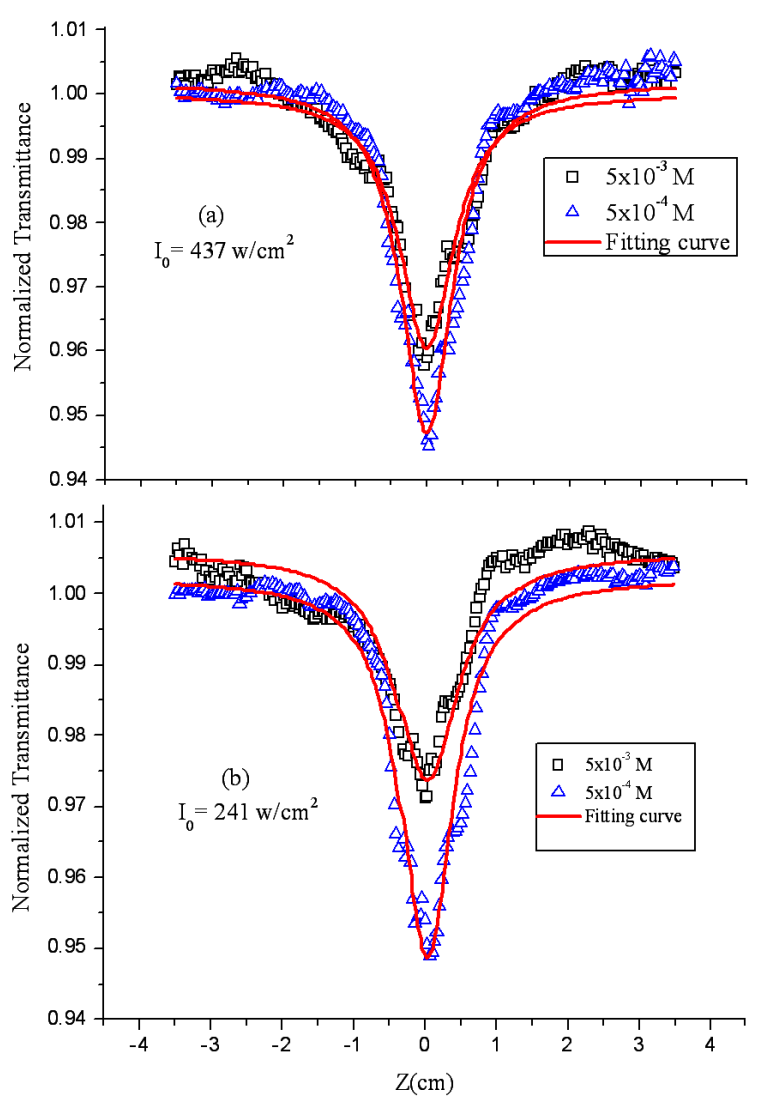

Fig. 4. Open-aperture $Z$-scan data of $1,1^{\prime}$-methylenedipyridinium pentacynidonitrosoferrate(II)pentahydrate in water for input intensities of (a) $I_{0}=437 \mathrm{~W} / \mathrm{cm}^{2}$ and (b) $I_{0}=241 \mathrm{~W} / \mathrm{cm}^{2}$ at two concentrations of $5 \times 10^{-3} \mathrm{M}$ and $5 \times 10^{-4} \mathrm{M}$.

of $I_{0}=437 \mathrm{~W} / \mathrm{cm}^{2}$ and $241 \mathrm{~W} / \mathrm{cm}^{2}$ for the two concentrations.

The open-aperture and closed-aperture of the $Z$-scan transmittance curves of the samples solutions were shown in Figs. 4 and 5, respectively. In the open-aperture $Z$-scan measurements $(S=1)$, when the sample is moved from negative $z$ to focus $(z=0)$, initially the laser beam

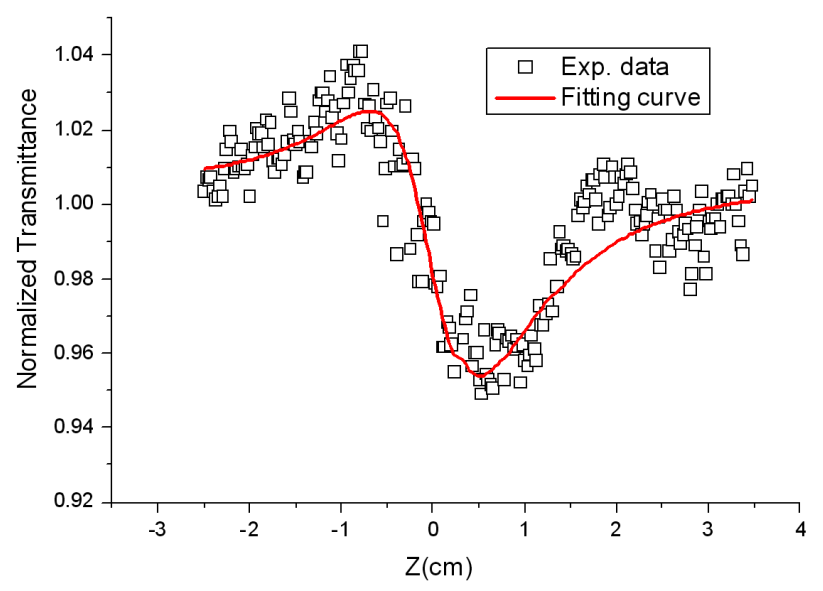

Fig. 5. Closed-aperture $Z$-scan data of $1,1^{\prime}$-methylenedipyridinium pentacynidonitrosoferrate(II)pentahydrate in water for $I_{0}=437 \mathrm{~W} / \mathrm{cm}^{2}$ at concentration of $5 \times 10^{-3} \mathrm{M}$.

intensity is low and the normalized transmittance is close to one. When the samples move closer to the focus, the laser beam intensity increases, leading to thermallensing effect in the sample media. The transmittance curves obtained are symmetric about focus for all sample concentrations, as shown in Fig. 4a,b. This characteristic indicates that our sample exhibits thermal induced nonlinearity due to the nonlinear absorption processes $[16,31,32]$. The nonlinear absorption in such materials arises from multiphoton absorption processes.

Figure 5 shows the closed-aperture data of the $1,1^{\prime}$ methylenedipyridinium pentacynidonitrosoferrate(II)pentahydrate in aqueous solution at concentration of $5 \times 10^{-3} \mathrm{M}$. The experimental data show that $1,1^{\prime}-$ methylenedipyridinium pentacynidonitrosoferrate(II)pentahydrate exhibits strong self-defocusing effect and negative sign of $n_{2}\left(n_{2}<0\right)$ as revealed in the obtained peak-valley shaped curve. The self-defocusing effect obtained in the studied sample is due to the local variation of the refractive index with the temperature $(\mathrm{d} n / \mathrm{d} T)$, and is attributed to a thermal nonlinearity resulting from the incident tightly focused CW laser beam. The localized absorption of the laser beam propagating through an absorbing solution medium produces a spatial distribution of the temperature in sample solution and, consequently, a spatial variation of the refractive index that acts as a thermal lens results in the phase distortion of the propagating beam [25, 33, 34]. The thermal effect has been discussed by Sheik-Bahae et al. $[15,16,35]$ in terms of temperature derivative of the index of refraction $(\mathrm{d} n / \mathrm{d} T)$ of the medium.

The laser beam induced nonlinear absorption and nonlinear refraction change in $Z$-scan measurements. The nonlinear absorption coefficient $\beta$ can be obtained from this open aperture $Z$-scan data by fitting the normalized transmittance data to the open aperture formula given as $[15,16]$ for $q_{0}<1$ : 


$$
T(z)=\sum_{m=0}^{\infty} \frac{\left(-q_{0}\right)^{m}}{(m+1)^{\frac{3}{2}}},
$$

where $q_{0}(z)$ is a parameter function of $I_{0}, L_{\text {eff }}$, and $\beta$ :

$$
q_{0}(z)=I_{0} L_{\text {eff }} \beta /\left(1+z^{2} / z_{0}^{2}\right),
$$

where $L_{\text {eff }}=\left[1-\exp \left(-\alpha_{0} L\right)\right] / \alpha_{0}$ is the effective thickness of the sample, $L$ is the thickness of the sample, $\alpha_{0}$ is the linear absorption coefficient.

Solving the summation (1) and for $\alpha_{0} \ll 1$ :

$$
T(z)=1-I_{0} L \beta /\left[2^{3 / 2}\left(1+z^{2} / z_{0}^{2}\right)\right],
$$

where $z_{0}=\pi \omega_{0}^{2} / \lambda$ is diffraction length of the beam, $\lambda$ is the laser wavelength, $\omega_{0}$ is the laser beam waist radius at the focal point and $I_{0}$ is the intensity of the laser beam at focus $z=0$. The solid lines in Fig. 4 are the fitting curves using Eq. (2), while the symbols are for the experimental data. The depth of the valley in open aperture $Z$-scans (Fig. 4) should be changed linearly after varying the intensity of the laser beam by nearly a factor of $2\left(I_{0}=241 \mathrm{~W} / \mathrm{cm}^{2}\right.$ and $\left.I_{0}=437 \mathrm{~W} / \mathrm{cm}^{2}\right)$. But, at the lower concentration of $5 \times 10^{-4} \mathrm{M}$ and $I_{0}=241 \mathrm{~W} / \mathrm{cm}^{2}$, this did not follow the linear behavior.

To determine the sign and magnitude of nonlinear refractive index $n_{2}$ of the studied sample, the normalized transmittance of closed aperture is given by [16, 35-37]:

$$
T(z, \Delta \varphi)=1-\frac{4 X}{\left(X^{2}+9\right)\left(X^{2}+1\right)} \Delta \varphi_{0},
$$

where $X=z / z_{0}$ and $\Delta \varphi_{0}$ is on-axis nonlinear phase shift. The normalized closed aperture $Z$-scan data are fitted with Eq. (3) to obtain $\Delta \varphi_{0}$ values. The nonlinear refractive index $n_{2}$ is related to $\Delta \varphi_{0}$ by Eq. (4):

$$
n_{2}=\frac{\lambda \Delta \varphi_{0}}{2 \pi I_{0} L_{\text {eff }}} .
$$

The variation between the normalized peak and valley transmission $T_{p-v}$ as function of $\Delta \varphi_{0}$ is given by

$$
\Delta T_{p-v}=0.406(1-S)^{0.25}\left|\Delta \varphi_{0}\right|,
$$

where $S$ is the linear aperture transmittance $S=1-$ $\exp \left(-2 r_{\mathrm{a}}^{2} / \omega_{\mathrm{a}}^{2}\right), r_{\mathrm{a}}$ is the radius of the aperture and $\omega_{\mathrm{a}}$ is radius of the laser beam waist.

Also, the nonlinear refractive index $n_{2}$ of the studied samples can be obtained experimentally from Fig. 5 by measuring $T_{p-v}$ and using Eqs. (4) and (5).

The calculated value of $n_{2}$ may have contributions of distinct origins, such as electronic $\left(n_{2}^{\mathrm{e}}\right)$ or thermal effects $\left(n_{2}^{\text {th }}\right)$. Hence, one can separate such contributions by the relation [38]:

$$
n_{2}=n_{2}^{\mathrm{e}}+n_{2}^{\mathrm{th}} \text {. }
$$

The thermal nonlinearity $n_{2}$ is related with the thermooptic coefficient $\mathrm{d} n / \mathrm{d} T$ by the

$$
\left(\frac{\mathrm{d} n}{\mathrm{~d} T}\right)=\frac{4 n_{2}^{\mathrm{th}} \kappa}{\alpha_{0} w_{0}^{2}},
$$

where $\kappa=0.609 \mathrm{~W} /(\mathrm{m} \mathrm{K})$ and $w_{0}$ are the thermal conductivity and the radius of beam waist, respectively. All the calculated values of the $n_{2}, \beta$, and the thermo-optic coefficient $\mathrm{d} n / \mathrm{d} T$ of our samples have been estimated and listed in Table I.

From the nonlinear refractive index $n_{2}$ and nonlinear absorption coefficient $\beta$, the real and imaginary parts of the third-order nonlinear optical susceptibility $\left(\chi^{3}\right)$ were calculated according to the following relations $[15,16]$ :

$$
\begin{aligned}
& \operatorname{Re} \chi^{3}(\mathrm{esu})=\left(10^{-4} \varepsilon_{0} c^{2} n_{0}^{2} / \pi\right) n_{2}\left[\mathrm{~cm}^{2} / \mathrm{W}\right], \\
& \operatorname{Im} \chi^{3}(\mathrm{esu})=\left(10^{-2} \varepsilon_{0} c^{2} n_{0}^{2} \lambda / 4 \pi^{2}\right) \beta[\mathrm{cm} / \mathrm{W}],
\end{aligned}
$$

where $\varepsilon_{0}$ is the vacuum permittivity and $c$ is the speed of light in vacuum.

Values of $\alpha_{0}$ and $n_{0}$ were measured for the studied sample and shown in Table II. Value of $\alpha_{0}$ was measured using a CW diode laser $(\lambda=635 \mathrm{~nm})$ at low incident power. The output power varies linearly with incident power, the transmittance obeyed the Beer law: $I=I_{0} \mathrm{e}^{-\alpha 0 L}$, where $I, I_{0}, \alpha_{0}$ and $L$ are the output power, the incident power, the linear absorption coefficient, and the sample path length, respectively. An Abbe refractometer is used for measurement of the linear refractive index $\left(n_{0}\right)$.

\section{TABLE II}

The measured values of linear refractive index $n_{0}$ and linear absorption coefficient $\alpha_{0}$ of $1,1^{\prime}$-methylenedipyridinium pentacynidonitrosoferrate(II)pentahydrate in aqueous solution at two concentrations of $5 \times 10^{-3} \mathrm{M}$ and $5 \times 10^{-4} \mathrm{M}$.

\begin{tabular}{c|c|c}
\hline \hline Concentrations & $\alpha_{0}\left[\mathrm{~cm}^{-1}\right]$ & $n_{0}$ \\
\hline $5 \times 10^{-3} \mathrm{M}$ & 0.67 & 1.3315 \\
$5 \times 10^{-4} \mathrm{M}$ & 0.46 & 1.3339
\end{tabular}

TABLE I

The calculated nonlinear optical parameters of $1,1^{\prime}$-methylenedipyridinium pentacynidonitrosoferrate(II)pentahydrate in aqueous solution for input intensities of $I_{0}=437 \mathrm{~W} / \mathrm{cm}^{2}$ and $241 \mathrm{~W} / \mathrm{cm}^{2}$ at two concentrations of $5 \times 10^{-3} \mathrm{M}$ and $5 \times 10^{-4} \mathrm{M}$.

\begin{tabular}{c|c|c|c|c|c|c}
\hline \hline Concentrations & $\begin{array}{c}\mathrm{d} n / \mathrm{d} T \\
{\left[\mu \mathrm{K}^{-1}\right]}\end{array}$ & $\begin{array}{c}I_{0} \\
{\left[\mathrm{~W} / \mathrm{cm}^{2}\right]}\end{array}$ & $\begin{array}{c}n_{2} \times 10^{-9} \\
{\left[\mathrm{~cm}^{2} / \mathrm{W}\right]}\end{array}$ & $\begin{array}{c}\beta \times 10^{-3} \\
{[\mathrm{~cm} / \mathrm{W}]}\end{array}$ & $\begin{array}{c}\operatorname{Re}\left(\chi^{3}\right) \times 10^{-7} \\
{[\mathrm{esu}]}\end{array}$ & $\begin{array}{c}\operatorname{Im}\left(\chi^{3}\right) \times 10^{-5} \\
{[\mathrm{esu}]}\end{array}$ \\
\hline \multirow{2}{*}{$5 \times 10^{-3} \mathrm{M}$} & 32.9 & 437 & 10.17 & 1.28 & 4.57 & 2.91 \\
& 241 & 64.8 & 20.03 & 1.87 & 9 & 4.25 \\
\hline \multirow{2}{*}{$5 \times 10^{-4} \mathrm{M}$} & 437 & 45.7 & 9.7 & 1.11 & 4.37 & 2.53 \\
& 47.6 & 241 & 10.1 & 1.89 & 4.55 & 4.31
\end{tabular}


Concentration dependence effect was investigated and shown in Fig. 4. It is indicated that the nonlinear absorption coefficient $(\beta)$ and nonlinear index of refraction $\left(n_{2}\right)$ are functions of concentrations of the samples. It has been found that the $\beta$ and $n_{2}$ values increase with an increase in concentration of the samples (Table I). This may be attributed to the fact that the number of molecules participating increases with an increase in concentration and more number of particles are thermally agitated, resulting in an increase in third-order optical nonlinearity [39]. From Table I it is clear that $\beta$ and $n_{2}$ decrease with an increase in the intensity which is produced as a result of thermal nonlinear process [40]. This indicates that the thermal effect becomes more prominent at lower beam intensity.

The obtained values of $\beta, n_{2},\left|\operatorname{Re} \chi^{3}\right|$ and $\left|\operatorname{Im} \chi^{3}\right|$ of the studied samples were determined using the fitting curves of the open/closed aperture measurements (Figs. 4 and 5) and Eqs. (1)-(7), and shown in Table I. The errors in estimating these values (Table I) arise from the uncertainties in the measuring of the focal spot size $( \pm 5 \%)$, linear refractive index $( \pm 0.3 \%)$, linear absorption coefficient $( \pm 5 \%)$ and the Rayleigh length $( \pm 4 \%)$. The maximum error in such measurements should be less than $5 \%$ for each parameter.

The reported values of $n_{2}$ and $\beta$ of the studied sample in Table I can be compared with recently reported values of $n_{2}$ and $\beta$ of different nonlinear optical materials with CW laser excitation [19, 41-44].

It can be seen that 1, $1^{\prime}$-methylenedipyridinium pentacynidonitrosoferrate(II)pentahydrate shows large thirdorder nonlinear optical properties because of the delocalized electronic states formed by the overlapping between $\pi \rightarrow \pi^{*}$ and $d$ orbitals $[19,44,45]$.

The experiments were repeated for the pure solvent to account for its contribution, but no significant measurable signals were produced in either the opened or the closed $Z$-scan traces. Also, it was found that the time employed for recording the $Z$-scan data in each plot does not affect the results for evaluating the nonlinear coefficients.

\section{Conclusion}

Synthesis, spectroscopic characterization, and the $Z$-scan measurements of 1, 1'-methylenedipyridinium pentacynidonitrosoferrate(II)pentahydrate, using a $\mathrm{CW}$ diode laser at $635 \mathrm{~nm}$ wavelength have been presented. Depending on the experimental results, values of $\alpha_{0}$, $n_{0}, n_{2}, \beta, \operatorname{Re} \chi^{3}, \operatorname{Im} \chi^{3}$, and the thermo-optic coefficient $\mathrm{d} n / \mathrm{d} T$ are calculated, and they are affected by the nature of attached ligand. It can be concluded that the combination of organic dication (with delocalized $\pi$-electron system) and inorganic anion (with $\pi$-electron system and $d$ orbitals) in 1, 1'-methylenedipyridinium pentacynidonitrosoferrate(II)pentahydrate gives good optical nonlinearity. The studied sample exhibits self-defocussing property with a negative value of nonlinear refractive index.

\section{Acknowledgments}

The authors would like to thank Prof. I. Othman, Director General, Prof. A.H. Al-Rayyes, head of the Radioisotope Department and Prof. M.K. Sabra for their encouragement and support, and also Mrs. K. Latifi and Mrs. H. Rihawi for their help in setting the experiment.

\section{References}

[1] S.H. Nalwa, S. Miyata (Eds.), Opt. Eng. 36, 2622 (1997).

[2] Characterization Techniques and Tabulations for Organic Nonlinear Optical Materials, Eds. M.G. Kuzyk, C.W. Dirk, Marcel Dekker, New York 1998.

[3] L.W. Tutt, T.F. Boggess, Prog. Quant. Electron. 17, 299 (1993).

[4] B. Chandrakantha, A.M. Isloor, R. Philip, M. Mohesh, P. Shetty, A.M. Vijesh, Bull. Mater. Sci. 34, 887 (2011).

[5] A.W. Allaf, M.D. Zidan, Laser Phys. 14, 1529 (2004).

[6] M.D. Zidan, M. Al-Ktaifani, A. Allahham, Opt. Laser Technol. 70, 45 (2015).

[7] S. Pramodini, P. Poornesh, Opt. Laser Technol. 63 , 114 (2014).

[8] T. Ceyhan, M. Yüksek, H. Gül Yağlığlu, B. Salih, M.K. Erbil, A. Elmalı, Ö. Bekaroğlu, Dalton Trans. 18, 2407 (2008).

[9] H.I. Elim, J. Ouyang, J. He, S.H. Goh, S.H. Tang, W. Ji, Chem. Phys. Lett. 369, 281 (2003).

[10] A.R. Kost, J.E. Jensen, R.O. Loufty, J.C. Wither, Appl. Phys. B 80, 281 (2005).

[11] H. Fan, X. Wang, Q. Ren, X. Zhao, G. Zhang, J. Chen, D. Xu, G. Yu, Z. Sun, Opt. Laser Technol. 42, 732 (2010).

[12] C. Yao, Y. Zhang, H. Yin, Q. Meng, C. Yu, J. Li, P. Yuan, Chem. Phys. Lett. 576, 35 (2013).

[13] Y. Ji, J. Zuo, L. Chen, Y. Tian, Y. Song, Y. Li, X. You, J. Phys. Chem. Solids 66, 207 (2005).

[14] Y. Cheng, Y. Mao, J. Liu, S. Feng, T. He, J. Mod. Opt. 54, 2763 (2007).

[15] M. Sheik-Bahae, A.A. Said, E.W. Van Stryland, Opt. Lett. 14, 955 (1989).

[16] M. Sheik-Bahae, A.A. Said, T. Wei, D.J. Hagan, E.W. Van Stryland, IEEE J. Quant. Electron. QE26760 (1990).

[17] K.B. Manjunatha, R. Dileep, G. Umesh, B. Ramachandra, Mater. Lett. 105, 173 (2013).

[18] T.C. Sabari Girisun, S. Dhanuskodi, G. Vinitha, Mater. Chem. Phys. 129, 9 (2011).

[19] H. Fan, Q. Ren, X. Wang, T. Li, J. Sun, G. Zhang, D. Xu, G. Yu, Z. Sun, Nat. Sci. 1, 136 (2009).

[20] Q. Ren, X.B. Sun, X.Q. Wang, G.H. Zhang, X.D. Yang, F.J. Zhang, H.I. Yang, Y.T. Chow, D. Xu, Appl. Phys. A 90, 685 (2008).

[21] J. Sun, W.F. Guo, X.Q. Wang, G.H. Zhang, X.B. Sun, L.Y. Zhu, Q. Ren, D. Xu, Opt. Commun. 280, 183 (2007).

[22] Q. Chang, X. Meng, Y. Song, H. Hou, Inorg. Chim. Acta 358, 2117 (2005). 
[23] H. Li, Z. Chen, L. Cheng, J. Liu, X. Chen, J. Li, Cryst. Growth Des. 8, 4355 (2008).

[24] B. Almarzoqi, A.V. George, N.S. Isaacs, Tetrahedron 42, 601 (1986).

[25] M.D. Zidan, A.W. Allaf, M.B. Alsous, A. Allahham, Opt. Laser Technol. 58, 128 (2014).

[26] W. Kemp, Organic Spectroscopy, 3rd ed., MacMillan Press Ltd, London 1991.

[27] C. De La Cruz, N. Sheppard, Spectrochim. Acta Part A Mol. Biomol. Spectrosc. 78, 7 (2011).

[28] M.M. Al-Ktaifani, M.K. Rukiah, Chem. Papers 65, 469 (2011).

[29] M.M. Al-Ktaifani, M.K. Rukiah, Chem. Papers 66, 1032 (2012).

[30] M.M. Al-Ktaifani, M.K. Rukiah, Acta Crystallogr. C 68, m246 (2012).

[31] I.A.H. Al-Saidi, S. Abdulkareem, J. Mater. Sci. Mater. Electron. 26, 2713 (2015).

[32] U. Majitha Parvin, M. Basheer Ahamed, Optik 126, 551 (2015).

[33] S. Zafar, Z.H. Khan, M.S. Khan, Spectrochim. Acta Part A Mol. Biomol. Spectrosc. 114, 164 (2013).

[34] F.Z. Henari, W.J. Blau, Proc. SPIE 2854, 174 (1996).

[35] F.L.S. Cuppo, A.M.F. Neto, S. L. Gömeze, P.P. Muhoray, J. Opt. Soc. Am. B 19, 1342 (2002).
[36] F.Z. Henari, P.S. Patil, Opt. Phot. J. 4, 182 (2014).

[37] M. Saravanan, T.C. Sabari Girisun, G. Vinitha, J. Mat. Sci. 51, 3289 (2016).

[38] R.F. Souza, M.A.R.C. Alencar, C.M. Nascimento, M.G.A. da Silva, M.R. Meneghetti, J.M. Hickmann, Proc. SPIE 6323, 63231T (2006).

[39] S. Pramodini, P. Poornesh, Opt. Laser Technol. 62, 12 (2014).

[40] Cheng-Bao Yao, Yun-Dong Zhang, Jin Li, Deng-Tai Chen, Hai-Tao Yin, Chang-Qiu Yu, Ping Yuan, Opt. Mater. 37, 80 (2014).

[41] S. Pramodini, P. Poornesh, Y.N. Sudhakar, M. Selva Kumar, Opt. Commun. 293, 125 (2013).

[42] K.K. Nagaraja, S. Pramodini, A. Santhosh Kumar, H.S. Nagaraja, P. Poornesh, D. Kekuda, Opt. Mater. 35, 431 (2013).

[43] S. Dhanuskodi, T.C. Sabari Girisun, S. Vinitha, Curr. Appl. Phys. 11, 860 (2011).

[44] M.D. Zidan, M. Alktaifani, A. Allahham, Optik 126, 1491 (2015).

[45] S. Wang, W. Huang, T. Zhang, H. Yang, Q. Gong, Y. Okuma, M. Horikiri, Y.F. Miura, Appl. Phys. Lett. 75, 1845 (1999) 